\title{
Artikel
}

Gabriele Dürbeck

\section{Narrative des Anthropozän - Systematisierung eines interdisziplinären Diskurses}


Abstract: The Anthropocene concept originates from earth system sciences and conceptualizes humanity as a planetary geophysical force. It links current action-oriented time horizons to Earth historical deep time and implies non-separability of natures-cultures. The Anthropocene concept has resonated in debates in natural and social sciences, the humanities and the broader public, serving as an inter- and transdisciplinary bridging concept. Based on an analysis of numerous texts from multiple scientific disciplines and media, this contribution distinguishes five narratives of the Anthropocene: the disaster narrative, the court narrative, the Great Transformation narrative, the (bio-)technological and the interdependence narrative. The five narratives articulate very different perspectives and experiences and transport divergent political, economic, ethical and anthropological values and interests; this is also shown in alternative conceptualizations such as Eurocene, Technocene, Capitalocene or Plantationocene. The analysis reveals that the narratives share significant structural characteristics concerning story, plot, protagonists, spatial and temporal structure and action-oriented emplotment which together can be referred to a meta-narrative of the Anthropocene. Since the partly overlapping, partly contradictory narratives compete for legitimation and dominance in science and the broader public, the findings raise the question whether this struggle will stabilize or undermine the Anthropocene meta-narrative in the long run.

Keywords: disaster narrative; court narrative; the Great Transformation narrative; the (bio-)technological narrative; the posthumanistic narrative

Secondary Keywords: Anthropocene as a metanarrative; emplotment; overlapping and competing narratives

Prof. Dr. Gabriele Dürbeck, Fakultät für Geistes- und Kulturwissenschaften (Fakultät III), Fach Germanistik, Universität Vechta, Driverstr. 22-26, 49377 Vechta, Email: gabriele.duerbeck@uni-vechta.de.

\section{Einleitung}

In der Gründungsausgabe der Kulturwissenschaftlichen Zeitschrift von 2016 hat Aleida Assmann betont, dass die zentrale Aufgabe der Kulturwissenschaften nicht nur in der "Selbstbeobachtung und Selbstthematisierung der Kultur" bestehe, sondern auch darin, mit der permanenten Veränderung der Lebenswelt, an der sie teilhaben, Schritt zu halten, brisante gesellschaftliche Fragen zu erforschen und damit zur "kulturellen Selbsttransformation" beizutragen (Assmann 2016: 47). Zweifellos stellt die kritische Reflexion des Anthropozän-Diskurses, der sich seit der Jahrtausendwende in den unterschiedlichsten Wissenschaftsbereichen und durch die Medien rasant ausgebreitet hat, eine solche Aufgabe dar. Der Begriff des Anthropozän bezeichnet den dominanten Einfluss des Menschen auf die biogeochemischen Subsysteme des Erdsystems in globalem Maßstab (Crutzen/Stoermer
2000). Es soll das Holozän ablösen, die seit ca. 11.700 Jahren andauernde Warmzeit mit relativ stabilen Umweltbedingungen, durch welche die Entstehung und Entwicklung der menschlichen Zivilisation ermöglicht wurde. Die zunehmende Erderwärmung durch Treibhausgase, irreversible Schäden durch Rodungen von Regenwäldern und Schadstoffeinträge in die Gewässer, die Versauerung der Meere, umweltschädlicher Plastikmüll in den Ozeanen sowie massiver Verlust von Biodiversität gehen auf tiefgreifende menschliche Veränderungen der Biosphäre auf planetarer Ebene zurück (vgl. Steffen et al. 2015).

Der Anthropozän-Diskurs hat eine Karriere in verschiedenen Wissenschaftsbereichen von der Geologie und den Umweltsystemwissenschaften, über die Science and Technology Studies, Sozialökonomie, Rechts-, Sozial- und Politikwissenschaften bis in die Archäologie und Geschichte, Philosophie und Theologie sowie Literatur- und Kulturwissenschaften angetreten. Dadurch ist ein schwer überschaubares Diskursgeflecht ent- 
standen. Die Idee einer neuen Erdepoche ist auch durch die Medien und die popularisierende Wissenschaftskommunikation (Schwägerl 2012; Leinfelder 2015) in eine breitere Öffentlichkeit vermittelt worden. Die britische Wochenzeitschrift The Economist titelte im Mai 2011: "Welcome to the Anthropocene" und betonte, dass "die Menschen den Planeten verändert haben und von nun an ihre Form, über ihn zu denken, ändern müssen"." 2013-2014 fand im Haus der Kulturen der Welt in Berlin unter Crutzens Schirmherrschaft ein groß angelegtes internationales und transdisziplinäres „Anthropozän-Projekt" statt, das mit dem Nachfolgeprojekt "Technosphere" (20152019) und den „Anthropocene Lectures" (20172018) fortgeführt wurde. Ebenfalls mit dem Titel "Willkommen im Anthropozän" warb eine Sonderausstellung im Deutschen Museum München (2014-2016), die mit einem vielbeachteten Katalog "Unsere Verantwortung für die Zukunft der Erde" ins Zentrum stellte (Möller et al. 2015).

Die Anthropozän-Hypothese mit der Idee eines vom Menschen geprägten Erdsystems stellt die kategorische Unterscheidung zwischen Natur und Kultur infrage und verlangt nach einer Neubestimmung der Stellung des Menschen als Bestandteil der lebendigen Welt. Obwohl das Anthropozän ursprünglich als geologisches Konzept eingeführt wurde, hat es sich in einem weiteren Verständnis rasch auch zu einem "kulturellen Konzept" (Trischler 2016a: 269; 2016b: 318-322) entwickelt, das weiterexistieren wird, auch wenn sich die geologische Anthropozän-Hypothese nicht bestätigen lassen sollte

Erkenntnisleitend für die folgenden Überlegungen ist die These, dass das Konzept des Anthropozän eine inhärente narrative Struktur hat, sodass es durch das Medium der Erzählung neuen Deutungen der conditio humana in unserer Zeit aufzeigt. Mit dem Anthropozän-Diskurs sind unterschiedliche Narrative verbunden, die zum Teil kontroverse philosophische, ökonomische, technologische, sozialökologische und ethische Interpretationen der Rolle des Menschen als geologischer Kraft und seiner Verbindung zur nicht-menschlichen Umwelt enthalten. Die Leitfrage dieses Beitrags ist, ob und inwiefern von einem Metanarrativ des Anthropozän in dem Sinne gesprochen werden kann, dass

1 http://www.economist.com/node/18744401 (abgerufen am 27.06.2017). die verschiedenen Anthropozän-Narrative im Hinblick auf Plot, Story, Protagonisten, räumliche und zeitliche Struktur sowie handlungsorientierende Sinnstiftungen gemeinsame Strukturmerkmale aufweisen und sich insofern auf das Metanarrativ beziehen lassen.

Der erste Abschnitt stellt das Anthropozän als inter- und transdisziplinäres Brückenkonzept vor und geht auf die Frage der Periodisierung der neuen Erdepoche ein. Danach werden der Begriff des Narrativs und dessen Funktionen in wissenschaftlichen Diskursen erläutert. Der dritte Abschnitt entwickelt eine heuristische Unterscheidung von fünf Narrativen des Anthropozän, um den bereits relativ ausdifferenzierten Diskurs in den Wissenschaften und den medialen Öffentlichkeiten zu systematisieren und gleichzeitig zu verdeutlichen, wie das Anthropozän-Konzept eine neuartige interdisziplinäre Zusammenarbeit von Natur-, Sozial-, Geistes- und Kulturwissenschaften in Gang gesetzt hat. Am Ende werden die unterschiedlichen Perspektiven der Narrative zusammengeführt und deren gemeinsamer Bezugspunkt wird verdeutlicht. Ein Ausblick schließt den Beitrag ab.

\section{Das Konzept des Anthropozän}

Im Jahr 2000 haben der Atmosphärenchemiker Paul Crutzen und der Limnologe Eugene F. Stoermer den Begriff des Anthropozän für eine neue erdgeschichtliche Epoche, die durch die dominante geophysikalische Macht des anthropos bestimmt sei, in die geologische und umweltwissenschaftliche Debatte eingeführt. ${ }^{2}$ Die Hypothese ist in der Folge laufend weiterentwickelt und bald

2 Etliche Vorläufer-Konzepte wie etwa "era anthropozoica" (Stoppani 1873: 163), ,Noosphäre' (Vladimir I. Vernadsky; Teilhard de Chardin), , anthropogene Ära' (Alexei P. Pavlov), ,Anthropozoikum' (Hubert Markl) oder "anthrocene" (Revkin 1992: 55) umschreiben bereits menschliche Einflüsse auf das planetare Ökosystem mit jeweils unterschiedlicher Schwerpunktsetzung und Ausgangsprämisse (vgl. http://www.astrobio.net/interview/the-anthropocene-humankind-as-a-turning-point-for-earth/ (abgerufen am 29.06.2017) und Trischler 2016b: 311). Demgegenüber betonen Hamilton/Grinevald (2015: 60f.) aber das Neuartige des Anthropozän-Konzepts, das erstmals in den Erdsystemwissenschaften fundiert sei. 
in die öffentliche Debatte hineingetragen worden (vgl. Crutzen/Stoermer 2000; Crutzen 2002; Crutzen/Steffen 2003; Steffen et al. 2003 und 2007; Zalasiewicz et al. 2010; Crutzen/Schwägerl 2011 u.v.a.). Seit 2009 berät die 38-köpfige Arbeitsgruppe der International Commission on Stratigraphy (ICS) der Geological Society London unter der Leitung von Jan Zalasiewicz ${ }^{3}$ darüber, eine neue geologische Epoche auszurufen, und auf dem 35. Kongress der Geological Society Ende August 2016 wurde schließlich der formale Grundstein dafür gelegt (mit 30 zu 3 Stimmen bei 2 Enthaltungen) (vgl. z.B. Carrington 2016).

Bemerkenswert ist, dass das Anthropozän-Konzept durchgehend als ein Narrativ präsentiert wird, als eine Geschichte mit Protagonisten, einer Ereigniskette, einem Plot mit Ursache-Wirkungs-Verhältnissen (vgl. Heise 2013: 21) und darüber hinaus mit einer spezifischen räumlichen und zeitlichen Struktur, die zusammen der Sinnstiftung dienen. Der Protagonist ist ungewöhnlich, nämlich die gesamte Menschheit als geophysikalische Kraft, die so tiefgreifende Spuren auf dem Planeten hinterlässt, dass sie auch nach "Hundertausenden von Jahren", etwa durch radioaktive Isotope oder $\mathrm{CO}_{2}$-Ablagerungen, noch in den Sedimenten nachweisbar sein werden (Renn/ Scherer 2015: 9; Masco 2010). Es entsteht eine "dialektische Konstellation" der Verantwortung für "eine schier endlos ferne Zukunft [...], ohne zu wissen, wie [die Menschheit] dieser Verantwortung gerecht werden kann" (Trischler 2016a: 277). Das Konzept der "planetary boundaries" (Rockström et al. 2009: 472) entwirft die Perspektive eines "safe operating space for humanity", dessen Parameter nicht überschritten werden dürfen. Die Idee der Unerschöpflichkeit der natürlichen Ressourcen, von der die gesamte Modernisierung, der Glaube an Fortschritt und technologische Entwicklung ausging, ist fundamental erschüttert (Malkmus 2015: 197); durch die Gefährdung des Planeten werde sich der Mensch seiner Endlichkeit bewusst. Angesichts des menschlichen Eingreifens wird die kulturell eingeschriebene Dichotomie von Natur und Kultur fundamental infrage gestellt. Nach Berechnungen des Kulturgeographen Erle C. Ellis sind mittlerweile mindestens $75 \%$ der bewohnbaren Erdoberfläche von Menschen über-

3 https://quaternary.stratigraphy.org/workinggroups/anthropocene/ (abgerufen am 29.06.2017). formte Natur, die er - abgeleitet von Biomen, ökologischen Großlebensräumen - als "Anthrome" bezeichnet (Ellis/Ramankutty 2008: 445). Demnach ist ,Natur' mittlerweile in großem und planetarem Maßstab anthropogene, eine vom Menschen kulturell und technisch überformte Natur. Schon vor dem Anthropozän-Diskurs sprach Bill McKibben vom "End of Nature" (1989) und aus posthumanistischer Sicht prägte Donna Haraway (2003: 2) für die enge Wechselbezüglichkeit von Natur und Kultur den sinnfälligen Begriff der „NatureCulture".

Das Anthropozän erscheint damit als ein Narrativ, das (a) die Menschheit als geophysikalische Kraft begreift, (b) eine planetarische Perspektive auf die globale Umweltkrise wirft, (c) eine tiefenzeitliche Zeitdimension aufweist, (d) eine enge Wechselbeziehung, d.h. Nicht-Trennbarkeit von Natur und Kultur annimmt und (e) daraus eine ethische Verantwortung des Menschen für das Erdsystem ableitet.

Bemerkenswert ist, dass sich in nahezu jeder Publikation eine Bemerkung zum Beginn der neuen geologischen Epoche findet und unterschiedliche Periodisierungen als Ausgangspunkt für die Differenzierung verschiedener Anthropozän-Narrative dienen. Bislang wenig verbreitet ist die Auffassung, das Anthropozän bestehe bereits seit dem Neolithikum, obgleich hier bereits menschliche Zivilisationstätigkeit und deutliche Veränderungen der physischen Natur beobachtbar sind. Dagegen sehen viele Vertreter der Anthropozän-Hypothese gute Gründe, die Erfindung der Dampfmaschine durch James Watt um 1784 (bzw. 1763, vgl. Dukes 2011) und die dadurch ausgelöste industrielle Revolution als Beginn der neuen Erdepoche anzusetzen (Crutzen 2002; Steffen et al. 2007 u.v.a). Als weiterer Marker wurde in einem Artikel in Nature das Jahr 1610 vorgeschlagen (vgl. Lewis/Maslin 2015), da sich bereits 100 Jahre nach Ankunft der Europäer massive sichtbare Veränderungen Nord- und Südamerikas zeigten; aus ähnlichem Grund spricht Donna Haraway (2015: 159) auch von einem "Plantationocene". In den letzten Jahren hat sich jedoch zunehmend die Auffassung verbreitet, dass die Freisetzung von Radionukliden infolge des Tests und des Einsatzes von Atombomben seit 1945 der zuverlässigste Marker sei. Wichtig bei der Festlegung einer neuen Zeitgrenze ist für die Geologie die globale Nachweisbarkeit einer markanten Veränderung in den Strata, den 
Gesteinsschichten und -sedimenten. Eine maßgebliche stratigraphische Methode ist die Bestimmung des "Golden Spike", also des genauen Zeitpunkts einer solchen Änderung. Dieser wird mit der ersten Detonation der Trinity-A-Bombe in New Mexico am 16. Juli 1945 und dem „Eintritt ins Nuklearzeitalter" (Zalasiewicz 2015: 164 und 176) bestimmt, in deren Folge sich der radioaktive FallOut schnell über den ganzen Erdball verbreitet hat. Zusätzlich sind aber, so das Ergebnis der Anthropozän-Arbeitsgruppe, viele weitere deutliche Signale des dauerhaften menschlichen Einflusses in den Sedimenten nachweisbar (Zalasiewicz 2015: 172-174), die unter dem Begriff der ,Great Acceleration' gefasst und durch Berechnungen mit ,Hockeyschlägerkurven' verdeutlicht werden (z.B. Steffen et al. 2011 und 2015). Dazu gehören vor allem der Anstieg von Stickstoff-Isotopen durch den weltweiten Gebrauch von Düngemitteln, der Anstieg des $\mathrm{CO}_{2}$-Gehalts in der Erdatmosphäre mit Ablagerungen selbst in den Polregionen, die weltweite Ausbreitung von Aluminium, Schwermetallen, Technofossilien und Plastik, besonders in der Form von Mikroplastikteilchen, die auch in die Nahrungskette gelangen, sowie die signifikante Zunahme von Arteninvasionen einerseits sowie massivem Artensterben andererseits.

Als am 29. August 2016 in diversen Zeitungen und Plattformen das lang erwartete Ergebnis der Arbeitsgruppe auf dem erwähnten 35. Internationalen Kongress der Geological Society mit den Worten verkündet wurde: "The Anthropocene is here" ${ }^{4}$ stach die Durchbruchsrhetorik ins Auge, in welcher der Akt der Benennung der neuen geologischen Epoche mindestens genauso wichtig erschien wie die wissenschaftliche Bestimmung selbst. Bei der Diskussion um die Durchsetzung des Anthropozän geht es damit auch um wissenschaftliche Reputation, Deutungshoheit und das Abstecken von Claims, die in den Narrativen transportiert werden. Die Bedeutung der Anthropozän-Hypothese wird mit den großen wissenschaftlichen Paradigmenwechseln wie Darwins Evolutionstheorie (Steffen et al. 2011: 862) oder

4 Vgl. z.B. http://time.com/4470514/anthropocene-geological-epoch-earth-scientists/ (abgerufen am 29.08.2016); http:// www.thehindu.com/sci-tech/science/The-Anthropocene-is-here-propose-scientists/article15618977.ece; http://www.ecologise.in/2016/08/30/the-anthropocene-is-here-scientists-declare-dawn-of-human-influenced-era/ (abgerufen am 30.06.2017). der von Galilei ausgelösten Kontroverse der Stellung des Menschen im Universum (Latour 2014: 3f.) parallelisiert.

Durch die Deklaration des neuen Erdzeitalters verschiebt sich zudem die Hierarchie der Disziplinen: Die wichtigsten Impulse für wissenschaftliche Innovation, aber auch für die Erklärung der Gegenwart, gehen derzeit von den Geo-, Klimaund Umweltwissenschaften aus, sie avancieren zu den neuen Leitwissenschaften, ${ }^{5}$ selbst bei Aufgaben, die traditionell eher geisteswissenschaftlichen Disziplinen zugedacht waren. Dass die genannten Datierungen keineswegs wert- und ideologiefrei sind, schlägt sich auch in deren Diskussion nieder. Würde das Anthropozän im Neolithikum beginnen, wofür beispielsweise Archäologen votieren (Balter 2013), hätte dies wohl kaum eine Auswirkung auf unser heutiges Denken und Handeln. Die Datierung auf 1610 lenkt den Blick auf die koloniale Unterwerfung von amerikanischen Ureinwohnern und die Ausbeutung ihres Landes, die durch die postkoloniale Forschung mittlerweile stärker ins Bewusstsein gerückt ist, aber nicht das Kriterium der global-planetarischen und tiefenzeitlichen Dimension erfüllt. Der Beginn mit der Erfindung der Dampfmaschine könnte zum einen zu einer Kritik des aufklärerischen Wissenschaftsparadigmas führen, das die Natur, in den [Kantischen] Richtstuhl der Vernunft spannt' (Scherer 2015: 106), zum anderen zu einer Kritik des Marktliberalismus, v.a. angelsächsischer Prägung (vgl. Oreskes/Conway 2014: 35-49), was für etliche Kritiker des Anthropozän längst an der Zeit sei (z.B. Dibley 2012). Demgegenüber lässt sich mit der Festlegung des, Golden Spike' auf die Zeit um 1950 „politischer Druck" ausüben (Rauchhaupt 2015), wie er trotz der anhaltenden Diskussionen zu den "Grenzen des Wachstums" (1972), der seit dem Brundlandt-Bericht 1987 und dem Erdgipfel von Rio 1992 virulenten Nachhaltigkeitsdebatte oder den jährlich stattfindenden UN-Klimaschutzkonferenzen bislang nur in einzelnen Industrieund Schwellenländern erzeugt werden konnte. So wird mit der Datierung des Anthropozän auf die Zeit der Hochindustrialisierung bzw. der ,Großen Beschleunigung' mit entsprechendem Anstieg der Treibhausgase und anderer beunruhigender ,Erd-

5 Horn und Schnyder (2016: 9) sprechen von der Klimatologie als neuer Leitwissenschaft, Bonneuil (2016: 17) nimmt eine geologische Wende (geological turn) an. 
systemtrends' (Steffen et al. 2015, Abb. 3) wiederholt der Ruf nach einem verantwortungsvollen Handeln gekoppelt: "Planetary stewardship has yet to emerge" (Steffen et al. 2015: 94). Das Narrativ wird also mit einem ethischen Auftrag, wenn nicht sogar mit einer politischen Agenda verbunden.

Zudem sind auf einer allgemeinen Ebene zwei grundlegend konträre Deutungen der Idee des Menschen als geophysikalischer Handlungsmacht zu unterscheiden. Angesichts der weltweiten Schäden betrachten die einen das Anthropozän pessimistisch als "Summe der ökologischen Frevel" (vgl. Leinfelder et al. 2012: 15) und den Menschen als Zerstörer, als ,Parasit' unseres Planeten (Krause 2014). Andere vertreten eine optimistische oder zumindest pragmatische Perspektive, wonach die Menschheit nunmehr ihr Schicksal selbst in der Hand habe und ,Gestalterin' der Erde sei. Der Kritik, etliche ,Anthropozäniker' verträten eine bloße Managementperspektive, wurde die Rede von "a good, or at least a better, Anthropocene" (Ellis 2011) entgegengestellt, da es keine Alternative zur Übernahme planetarischer Verantwortung (planetary stewardship) gebe. Aber auch hier gehen die Meinungen weit auseinander: Während die Vertreter eines good Anthropocene die neuen technologischen und organisatorischen Handlungsmöglichkeiten für die Gestaltung einer besseren Zukunft des Menschen betonen und darin "an amazing opportunity" (Ellis 2011) sehen, diagnostizieren die Skeptiker "a techno-industrial hybris" (Hamilton 2015: 233), d.h. eine neuerliche, schon längst als überwunden geglaubte Zentralstellung des Menschen, und beklagen eine fehlende Einsicht in die "unintendierten Nebenfolgen" menschlichen Eingreifens (z.B. Heise 2013: 21; Scherer 2015: 106). Damit sind unterschiedliche Interpretationen angesprochen, die sich an die Anthropozän-Hypothese anschließen und die distinkte, zum Teil gegenläufige Narrative ausgebildet haben.

\section{Zum Konzept des Narrativs}

Bevor die einzelnen Narrative des Anthropozän dargelegt werden, sollen das Konzept des Narrativs und seine Funktionen erläutert werden. Den Literaturwissenschaftlerinnen Astrid Erll und Simone Roggendorf (2002: 77) zufolge sind Narrative „zentrale kulturelle Ausdrucksformen, die wesentlich zur Selbstdeutung und Sinnstiftung einer Kultur beitragen". Narrative lassen sich daher "im weitesten Sinne als historisch wandelbare Phänomene kollektiver Wirklichkeitserzeugung, Sinnstiftung und zwischenmenschlicher Verständigung" betrachten (Erll/Roggendorf 2002: 79). Diese Bestimmung geht vom Erzählen als anthropologischer Konstante aus, wobei der Mensch als "homo narrans" (Fisher 1984: 6) bzw. "storytelling animal" (MacIntyre 1984: 216) gilt.

Ein ganz ähnliches Verständnis von Narrativen findet sich in Albrecht Koschorkes Studie zu den Grundzügen einer Allgemeinen Erzäh/theorie (2012). Darin setzt er die Ubiquität des Phänomens Erzählen voraus, sei es in der Alltagskommunikation, in der Literatur oder den Wissenschaften bis zu den "master narratives", in denen sich ganze Gesellschaften mit ihren "Gründungsmythen und Lebenslügen" wiedererkennen (Koschorke 2012: 19). Indem das Erzählen "eine Sonderwelt neben der wirklichen Welt hervorbringt" und "in die gesellschaftliche Praxis hineinwirkt", ist es „Organon einer unablässigen kulturellen Selbsttransformation" (Koschorke 2012: 25). ${ }^{6}$ Wie Aleida Assmann festgestellt hat, geht es Koschorke weniger um das Erzählen als kreativem Sprechakt oder poetischer Textur von literarischen Texten, als um das Narrativ im Sinne einer "abstrakten Struktur, die zugleich eine Form der Sinngebung ist, in die Handlungen, politische Ziele, Geschichtsbilder und menschliche Erfahrungen" gefasst werden; Narrative sind demnach "der rote Faden, der eine klar definierte Auswahl heterogener Ereignisse zusammenhält und ihnen mit Anfang, Mitte und Ende zugleich Struktur, Sinn und Ziel verleiht" (Assmann 2016: 45f.). In dieser Hinsicht hatte bereits der Historiker Hayden White das Konzept des „emplotment" eingeführt, also der "narrativen Modellierung" (White 1991: 21-25), die "heterogene Erfahrungs- und Wissenselemente zu einer mehr und weniger einheitlichen Ganzheit [verknüpft], die eine kontinuierliche Abfolge von Ereignissen suggeriert"; sie dient der Sinnstiftung und "Kontingenzreduktion" (vgl. Neumann 2013: 553). Zudem bieten Narrative "Orientierung" und "simulieren [...] Erkenntnis", sollten aber nicht verabsolutiert,

6 Koschorke (2012: 19) folgert, der Begriff der Erzählung sei „zu einer der transdisziplinär erfolgreichsten und expansionsfreudigsten literaturwissenschaftlichen Kategorien geworden", was zu einem narrative turn in den verschiedenen Wissenschaften geführt habe. 
sondern lediglich als „Verlegenheitslösungen mit praktischer Notwendigkeit und unbezweifelbarer Legitimität" angesehen werden, um „Möglichkeiten (des Verstehens und Handelns) gleichermaßen offen zu halten" (Becker-Lindenthal 2017: 165f.).

Wenn die Anthropozän-Hypothese nun mit bestimmten Narrativen verbunden wird, hat dies strategische und reflexive Aspekte. In strategischer Hinsicht haben einzelne Narrative des Anthropozän die Funktion, angesichts der enormen Komplexität von Ursache-Wirkungs-Zusammenhängen, Wechselwirkungen, Rückkopplungsschleifen und nicht-intendierten Nebenwirkungen der menschlichen Aktivitäten und der dadurch ausgelösten beunruhigenden Erdsystemtrends seit 1950 (vgl. Steffen et al. 2015) eine sinnvolle und handlungsleitende Interpretation der Situation zu vermitteln. In reflexiver Hinsicht stellen Anthropozän-Narrative etablierte Positionierungen des Menschen infrage. Außerdem werden im Anthropozän-Diskurs Zusammenhänge artikuliert, die sich einer klaren Lokalisierbarkeit und Sichtbarkeit entziehen und nicht auf herkömmliche Weise repräsentierbar sind, sodass gängige Sinngefüge nicht mehr greifen (vgl. Clark 2015: xi) und neue Narrative zur Sinnkonstituierung und -vermittung notwendig sind. Einige LiteraturwissenschaftlerInnen sehen daher insbesondere den Ecocriticism und die Environmental Humanities in der Pflicht, neue Repräsentationsformen und Rezeptionsverfahren zum Verständnis der hochkomplexen Zusammenhänge des Anthropozän zu erschließen, was in der Folge die Frage nach einer eigenen Poetik des Anthropozän aufwirft (s.u. Abschnitt 4.5). Neuere Ansätze in dem sich herausbildenden Feld der Econarratology (James 2015) behandeln stärker als der oft eher themenorientierte Ecocriticism Fragen der Form, der Erzählstrukturen und der Erzählstrategien. Als ökonarratologisch versteht sich auch die kritisch-produktive Auseinandersetzung mit Michel Serres' philosophischer Ökologie (z.B. Watkin 2015). Eine vertiefte Diskussion solch unterschiedlicher Ansätze der Econarratology ist im Rahmen dieses Artikels jedoch nicht möglich.

\section{Fünf Narrative des Anthropozän}

Eine systematische Literaturrecherche von mehr als 600 natur-, sozial- und kulturwissenschaft- lichen sowie publizistischen Veröffentlichungen zum Anthropozän seit 2000 hat ergeben, dass sich fünf Narrative unterscheiden lassen. ${ }^{7} 1$. das Katastrophen- bzw. Apokalypsenarrativ; 2. das Gerichtsnarrativ; 3. das Narrativ der ,Großen Transformation', 4. das (bio-)technologische Narrativ und schließlich 5 . das Interdependenz-Narrativ, das nach einer kritischen Antwort auf die Aufhebung der Natur-Kultur-Dichotomie sucht. ${ }^{8}$

\subsection{Das Katastrophen- bzw. Apokalypsenarrativ ${ }^{9}$}

Eine Reihe von Geistes-und KulturwissenschaftlerInnen hält das Ausmaß der Umweltzerstörung,

7 Mit dieser Systematisierung stellt der Beitrag erste Ergebnisse des im Januar 2017 angelaufenen DFG-Projekts „Narrative des Anthropozän in Wissenschaft und Literatur. Themen, Struktur und Poetik" vor, vgl. https://www.uni-vechta.de/uni/anthropozaen-projekt/. - Für zahlreiche Hinweise zu diesem Artikel danke ich Philip Hüpkes.

8 Die vorliegende Heuristik ist induktiv und parallel zu Christophe Bonneuils (2016) Unterscheidung von vier Narrativen entwickelt worden, wobei Bonneuil die einzelnen Narrative jedoch nicht auf ein Metanarrativ bezieht und m.E. auch zu wenig deren Überlappungen und konkurrierenden Geschichten berücksichtigt hat. Bonneuil (2016: 23) unterscheidet (1) ein im Anthropozän-Diskurs dominantes "naturalist narrative", welches das ,grand narrative' der Moderne vom Menschen als quasi-natürlicher Macht zu reproduzieren tendiere, worauf er einen sehr kritischen Blick wirft, von (2) dem „post-nature narrative” (23f.), das ausgehend von der Annahme vom Ende der Natur ein geokonstruktivistisches, technologisches und ökopragmatisches Projekt eines, good Anthropocene" verspreche; davon hebt er (3) das "eco-catastrophist narrative" (26f.) und das "eco-Marxist narrative" (27-29) ab. Auf Ähnlichkeiten und Unterschiede zwischen den beiden Heuristiken wird i.F. bei den einzelnen Abschnitten eingegangen. - Der vorliegende Ordnungsvorschlag unterscheidet sich zudem von einer weiteren Unterteilung, die Bonneuil und Fressoz in ihrem vielrezipierten Buch The Shock of the Anthropocene (2015) vorgeschlagen haben, nämlich eine historische Sequenzierung von sechs Subnarrativen des Anthropozän-Diskurses (Thermocene, Thanatocene, Phronocene, Agnotocene, Capitalocene, Polemocene), die bestimmte industrielle, technologische, ökonomische und militärische Entwicklungen und die Geschichte der ökologischen Bewegungen herausarbeitet. Bonneuil (2016) greift die 2015 entwickelte Unterteilung jedoch nur hinsichtlich des Kapitalozäns und des Aufklärungsnarrativs (später "naturalist narrative") auf. 9 Dieses Narrativ deckt sich weitgehend mit Bonneuils "eco-catastrophist narrative" (26f.), legt aber anderes Material zugrunde. 
insbesondere seit der Phase der Hochindustrialisierung, für so groß, dass, die Katastrophe' unabwendbar sei. Peter Sloterdiijk (2015: 36) spricht von einer "apokalyptischen Logik", da der Mensch durch die Einsicht in die Endlichkeit der natürlichen Ressourcen seine "kosmische [...] Unbesorgtheit" verloren habe. In eine ähnliche Richtung geht die Diagnose von Bernhard Malkmus (2015: 197), „das Zeitregime der Moderne, das paradoxerweise auf dem Glauben an die unversiegbare Regenerationsfähigkeit des Lebens auf der Erde" beruht, sei „zu Ende". "Dystopisten" sähen den "Technologiepark der Moderne" als "Käfig", der die Menschen "von wesentlichen regenerativen Kräften abschneide oder gar in die Selbstzerstörung führe" (Malkmus 2015: 183). Die westliche Zivilisation steuere auf einen "Kollaps" (Oreskes/Conway 2014) zu. Eva Horn (2014) spricht pointiert von der "Zukunft als Katastrophe", während Jürgen Renn und Bernd Scherer (2015: 15f.) "angesichts apokalyptischer Bedrohungen" "die Notwendigkeit des Handelns" konstatieren, die eine "Selbstreflexion" des "anthropozänen Denken[s]" voraussetze.

Naturwissenschaftliche Vertreter der Anthropozän-Hypothese sehen "the viability of contemporary civilization and perhaps even the future existence of Homo sapiens" vor allem dann gefährdet, wenn die Befürworter der ,Großen Beschleunigung' ungehindert weitermachen (Steffen et al. 2011: 862) und betonen die Möglichkeit, die Weichen im Rahmen der "planetary boundaries" (Rockström et al. 2009) neu zu justieren. Aufgrund der Great Acceleration lasse sich sogar von einem "new historical meta-narrative" sprechen, denn die Menschheitsgeschichte ist "marked by crises, regime shifts, disasters and constantly changing patterns of adjustments to limits and confines", also durch eine Abfolge von Phasen des Wachstums, des Zusammenbruchs und der Neuorganisation als einem "common pattern in the human past" (zit. n. Steffen et al. 2015: 94). Andere sehen aus diesem Muster jedoch keinen Ausweg: Die Philosophin Claire Colebrook etwa weist in ihrem Buch Death of the PostHuman (2014: 249) die Idee einer posthumanen Zukunft zurück, da die menschliche Spezies durch "Kurzsichtigkeit und Anthropozentrismus" geprägt sei und deshalb weder als „Grundlage" noch als „kritischer Hebel" im Anthropozän dienen könne.
In historischer Perspektive lässt sich für das Katastrophennarrativ eine Linie von dem innovativen Aufklärungsgeologen Georges Cuvier über Georg Friedrich Lichtenberg, Georg Büchner und Friedrich Nietzsche bis zu Ulrich Horstmann ziehen, die alle angesichts der Entdeckung der geologischen Tiefenzeit und der Abfolge von einschneidenden katastrophischen Ereignissen eine Marginalisierung des Menschen sehen und das Bild eines "menschenfeindlichen, verlassenen Planeten Erde" zeichnen (Braungart 2009: 55). Ein vielfach auffindbares Thema ist das Artensterben, so etwa in Elizabeth Kolberts mit dem Pulitzer-Preis ausgezeichneten Sachbuch The Sixth Extinction (2015). Auch Timothy Morton (2015: 258) hebt das sechste Massensterben als zentrales Merkmal des Anthropozän hervor. Die Geschichte des vom Menschen ausgelösten Verfalls und Untergangs der Natur (vgl. Heise 2016) konkretisiert sich in einer oft melancholischen Sprache oder einem "elegischen Narrativ" (Bühler 2016: 154). Eine in verschiedenen Wissenschaften und Essays vorkommende Metapher ist die des ,kranken Planeten'. Sie findet sich schon bei Schopenhauer (1973: 11), der das Bild einer ,von Schimmel überzogenen Erde' geprägt hat und damit einen "latenten Selbstekel des Homo sapiens" (Wenzel 2013) artikuliert. Und Nietzsche (2003: 164) klagt: "die Erde [...] hat eine Haut; und diese Haut hat Krankheiten: Eine dieser Krankheiten heisst zum Beispiel: Mensch".

Auch stellt sich die Frage, was von der Welt übrig bleibt, wenn der ,Titelheld' des neuen Erdzeitalters aussterben sollte. Aus der Perspektive der Zukunft lässt sich neben schriftlichen Dokumenten (Oreskes/Conway 2014) allein aufgrund der Ablagerungen in den Strata die menschliche Zivilisations- und Industrialisierungsgeschichte rekonstruieren. In dieser Hinsicht fragt der Paläobiologe Jan Zalasiewicz (2008): "what legacy will humans leave in the rocks?" Ein solcher archäologischer Blick aus der Zukunft fokussiert die materielle Seite des Planeten, an dem großskalige Prozesse abgelesen werden können. Damit wird der Begriff des ,Archivs' als Summe von textuellen und medialen Quellen zur Dokumentation historischer Entwicklungen (vgl. Boes/ Marshall 2014: 68) erweitert, in dem er z.B. auch auf die von Geologen gesammelten Eisbohrkerne oder Fossilien und spektakuläre Kno- 
chenfunde bezogen wird. Die Rede von geologischer Zeit bzw. Tiefenzeit würde dann nicht als Metapher betrachtet, sondern die geologischen Schichten werden selbst zu Medien, sodass sich nicht nur neue literatur- und kulturtheoretische, sondern auch medienarchäologische und -ökologische Fragen nach technischen und medialen Ablagerungen ergeben. In dieser Hinsicht meint Malkmus (2015: 196), Geologen seien nicht nur Semiotiker, sondern auch "Kulturwissenschaftler" bzw. Geologen betrieben Medienwissenschaft (Parikka 2015). Dieser Blick auf die eigene Kultur von einem antizipierten Ende her führt zur Verfremdung und erlaubt eine kritische Reflexion der Gegenwart. Für das Katastrophennarrativ sind damit die mahnende Appellfunktion und der kritische Impuls entscheidend, wie sie sich auch in der Literatur, v.a. in der Science Fiction, finden.

\subsection{Das Gerichtsnarrativ}

Das Gerichtsnarrativ ähnelt dem Katastrophennarrativ, indem ebenfalls die weitgehende Zerstörung des Planeten thematisiert wird. Doch steht hier die Frage nach der Verursachung und Haftbarkeit für die Schäden im Mittelpunkt. Wenn man annimmt, dass die Idee des Anthropozän eine "Botschaft von nahezu unüberbietbarer moralisch-politischer Dringlichkeit" ist (Sloterdijk 2015: 25), stellt sich die Frage der Schuld und der Verantwortung. Wer hat den Schaden angerichtet? Ist die ganze Menschheit auf die Anklagebank zu setzen? Wer ist der Richter? Nach welchen Normen ist über die Anklage zu entscheiden? Muss angesichts der weltweiten Disparitäten und Kausalitäten zwischen Tätern und Opfern unterschieden werden (vgl. Becker-Lindenthal 2017: 163)? Für viele Vertreter der Anthropozän-Hypothese steht zweifelsfrei fest, dass die Hauptverursacher die westlichen Industrienationen und ihre "technokratischen Elite[n]" sind, weshalb Peter Sloterdijk (2015: 27) auch von einem „Eurozän" oder einem "Technozän" spricht. Zudem wird gezeigt, dass die Auswirkungen die Armen im Globalen Süden ungleich härter treffen (Chakrabarty 2015: 151) und damit eine globale Diskrepanz der $\mathrm{CO}_{2}$-Emisionen und der Lastenverteilung des Klimawandels besteht (Althor et al. 2016). Diskurskritiker hingegen wenden die Anklage gegen das Anthropozän-Narrativ und seine Proponenten selbst und kritisieren den Begriff, der "von einer Minderheit (einer männlichen weißen Oberschicht)" geprägt sei (Becker-Lindenthal 2017: 158). Da die Industrialisierung an das neuzeitliche Kapitalsystem und westliche Märkte gebunden ist, sprechen darüber hinaus etliche auch vom "Kapitalozän" (z.B. Bonneuil/Fressoz 2015: 222-252; Moore 2016). ${ }^{10}$ Dabei werden die Ursachen der globalen Schäden im Kapitalismus als gesellschaftlich-wirtschaftlichem Ordnungssystem verortet, dessen Proponenten Schuld und Verantwortung tragen. Allein an diesen verschiedenen Benennungen wird deutlich, dass das Gerichtsnarrativ divergierende Geschichten ermöglicht.

Bei der Frage nach den Schuldigen und den erforderlichen politischen, technologischen, ökonomischen und sozialen Maßnahmen kommt hinzu, dass die Rede von der Menschheit ein Kollektivsubjekt voraussetzt, das als solches gar "kein handelnder Akteur" sein kann (Manemann 2014: 35). Es besteht die Gefahr, dass am Ende keiner verantwortlich ist (Feindt 2002). Wenn also Crutzen und Steffen (2003: 256) und weitere der Anthropozän-Arbeitsgruppe von "a responsible stewardship of the Earth System" sprechen, müsste gemäß dem multilateralen Rechtsprinzip, wie es in der UNFCCC verankert ist, genauer differenziert werden, wer verantwortlich ist und wer für die Schäden aufkommen soll. Deshalb haben Umweltaktivisten eine Differenzierung der "ProKopf-Emission" gefordert, die dem Prinzip einer "gemeinsame[n,] aber differenzierte[n] Verantwortung für die globale Erwärmung" und deren Bekämpfung entspreche (Chakrabarty 2015: 153). Die Rede vom Kollektivsubjekt könnte dazu dienen, "eigene, parteiische Interessen [...] zu verschleiern" (Chakrabarty 2015: 154) und wichtige Fragen der Ungleichheit zu übergehen ( $\mathrm{vgl}$. Malm/Hornborg 2014). In einem neueren Aufsatz geben darum Will Steffen et al. (2015: 91) zu bedenken: "treating the humans as a single, monolithic whole [...] ignores the fact that the Great Acceleration [...] has been almost entirely driven by a small fraction of the human population", wobei sie zwischen den von den OECD-Ländern, den BRICS-Staaten (Brasilien, Russland,

10 Das ,eco-Marxist narrative' nach Bonneuil (2016: 2729) sieht das Anthropozän als Ergebnis eines Widerspruchs des Kapitalismus, nämlich seiner Unfähigkeit der Pflege von Natur. Das Gerichtsnarrativ jedoch ist weiter gefasst. 
Indien, China, Südafrika) und den von anderen Ländern verursachten Umweltschäden unterscheiden. Der Vergleich rekurriert auf die sozioökonomischen Entwicklungen der verschiedenen Länder hinsichtlich Bevölkerungsentwicklung, Düngemittelverbrauch, Bau großer Staudämme, Wasserverbrauch, Papierherstellung, Transportsystemen und Telekommunikation und kann deutlich zeigen: "the most of the human imprint on the Earth System is coming from the OECD world" (Steffen et al. 2015: 91).

Wichtig in diesem Kontext ist ein Argument, das die Wissenschaftshistoriker Christophe Bonneuil und Jean-Baptiste Fressoz (2015: xiii, 72) herausgearbeitet haben: Die Erfinder der Anthropozän-Hypothese würden eine "story of awakening" erzählen, wonach wir (als Menschheit) die Natur unwillentlich zerstört hätten, aber jetzt die Erdsystemwissenschaften hätten, um unsere Fehler zu erkennen. Die ,Geschichte des Erwachens' impliziert die problematische Annahme, dass die Umweltzerstörung begann, bevor man ihre Konsequenzen habe wissen können, sodass auch hier die Frage der Verursachung durch die Industrieländer verschleiert werde. Die Annahme, Wissenschaftler, Technologen und frühere Generationen hätten nicht gewusst, was sie tun, weisen Bonneuil und Fressoz (2015: 76) als historisch falsch zurück: "the period between 1770 and 1830 was marked by a very acute awarenss of the interactions between nature and society". Sie verdeutlichen zudem die ideologische Funktion dieses Narrativs, wonach Wissenschaftler als die neuen Experten oder die neuen "Helden" (Bonneuil/Fressoz (2015: 79) installiert würden, die uns nun von Blindheit zur Erkenntnis führen könnten. Wegen des implizierten Heroismus kritisieren Bonneuil/ Fressoz (2015: 48, 93) die ,story of awakening' als "hegemonic" und "geocratic".

\subsection{Das Narrativ der Großen Transformation}

Im Narrativ der Großen Transformation ist ein Ausweg aus der globalen Umweltkrise noch möglich, sofern schnell gehandelt und der, business-as-usuar-Ansatz überwunden wird. Das Anthropozän wird als ethisch-politischer Auftrag verstanden, durch "[...] a responsible stewardship of the Earth System" (Crutzen/Steffen 2003: 256) eine lebensfähige Zukunft für die nächsten Generationen zu schaffen. Um dies zu erreichen, werden von Vertretern der Anthropozän-Hypothese zwei unterschiedliche Optionen diskutiert, eine integrierte technisch-ökologisch-ökonomisch-soziale Modernisierung und eine technozentrische, die besonders das (bio-)technologische Narrativ (s.u. Abschnitt 4.4) prägt; insofern sind beide auf eine tiefgreifende Transformation angelegt, doch mit unterschiedlichen Mitteln, weshalb die beiden Narrative hier heuristisch nochmals unterschieden werden.

Die Große Transformation soll durch eine Mischung aus "Verminderung" (mitigation) der Ursachen der Umweltzerstörung und "Maßnahmen der vernünftigen Anpassung" durch bessere Technologien und höhere Umwelteffizienz, notfalls auch durch verminderten Konsum, erreicht werden (Mastrandrea/Schneider 2011: 17f.; vgl. Steffen 2007: 619). Diese Maßnahmen greifen den Diskurs der ökologischen Modernisierung auf, für den in der Öffentlichkeit namhafte Personen wie etwa Hans Joachim Schellnhuber, Gründer des Instituts für Klimafolgen in Potsdam (PIK), langjähriges Mitglied des Weltklimarats und seit 2009 Vorsitzender des Wissenschaftlichen Beirats der Bundesregierung Globale Umweltveränderungen (WBGU), stehen. Ziel ist es, gemäß dem Vorsorgeprinzip mit möglichst effizienten und weitsichtigen Strategien die Schadensanfälligkeit und Verletzbarkeit einer sich rapide verändernden Umwelt zu minimieren und die technischen Innovationsprozesse auch in den Ländern des Globalen Südens nach dem Solidaritätsprinzip und dem "fair burden sharing" (Schellnhuber/ Huber 2013: 7) so zu gestalten, dass die ökologische Modernisierung ökonomisches Wachstum berücksichtigt und die Lasten dafür gleichmäßiger verteilt werden. Im WBGU-Gutachten (2011) „Welt im Wandel: Gesellschaftsvertrag für eine große Transformation" wurden diese und weitere Ziele insbesondere in den Handlungsfeldern Energiewende, Urbanisierung und Landnutzung für die EU detailliert ausformuliert."

11 Der Sachbuch-Comic mit dem sprechenden Titelbild "Kriegen wir die Kurve?" (2013) stellt eine Synthese des WBGU-Gutachtens (2011) "Welt im Wandel - Gesellschaftsvertrag für eine Große Transformation" (2011) dar, in dem prominente Vertreter der Klimawandel- und Anthropozän-Debatte wie Schellnhuber, Leggewie oder Messner als ,Helden' auftreten. 
Durch die Große Transformation soll die Funktionsfähigkeit der sozial-ökologischen Systeme stabilisiert werden. Dies erfordert im Diskurs des ,civic environmentalism' die Beteiligung der Zivilgesellschaft und eine weitere Demokratisierung, sodass globale Maßnahmen nicht nur von oben - bzw. erneut von den Industrienationen - verordnet, sondern zugleich in einer bottom-up-Perspektive lokale Handlungsspielräume erschlossen werden (Mastrandrea/Schneider 2011: 52f.). Bekannte Proponenten der Großen Transformation wie Harald Welzer oder Claus Leggewie, ehemaliger Direktor des Kulturwissenschaftlichen Instituts Essen und Mitglied des WBGU, halten einen Umbau zu einer "verantwortungsvollen und nachhaltigen Gesellschaft" bei einem radikalen "Kulturwandel" mit verändertem Konsumverhalten, Modernisierung der Demokratie und Bürgerbeteiligung für möglich (Leggewie/Welzer 2009: 230). Ein Dilemma ist dabei freilich die Verknüpfung von abstrakten Problemlagen mit konkretem Alltagshandeln.

Die normativen Zielsetzungen werden durch Metaphern und Bilder vermittelt, allen voran die Metapher des Gärtners oder sogar des „Weltgärtners", wonach die Umwelt nicht mehr als das Außen und das Fremde, sondern als "Uns-Welt" (Leinfelder 2013) betrachtet wird. Namhafte Vertreter dieser Perspektive sind der Geowissenschaftler Reinhold Leinfelder und der Wissenschaftsjournalist Christian Schwägerl (2012: 57). Dabei ist die Gartenmetaphorik keineswegs eindeutig. Während etwa bei der französischen Gartenkunst der Umgang mit der Natur "auf Aneignung und Naturbeherrschung ausgerichtet" ist, lässt sich für den englischen Landschaftsgarten von einer "Allianztechnik" (Böhme 1989: 85, 84) sprechen, also der Bearbeitung der Natur bei gleichzeitiger Anerkennung ihrer Selbsttätigkeit. Die Gartenmetapher findet sich auch in Schwägerls vielrezipiertem Buch Menschenzeit (2012), das in der Süddeutschen Zeitung wegen seiner "neue[n] Bilder und Erzählformen" gelobt wurde, womit eine optimistische Art und Weise gemeint ist, die Erde neu zu denken und "eine Lösung zu finden, die dem Anthropozän doch noch eine Wendung zum Guten geben kann" (Häntzschel et al. 2014). Am Ende von Menschenzeit entwirft der Autor ein Bild von der Menschheit als "gereifte Primaten" (Schwägerl 2012: 320), eine "höchst diverse, aber vernetzte Gemeinschaft von Erd- gärtnern" (349), die noch eine ",lange Zukunft" (323) vor sich habe; ausdrücklich ist hier auch von "Eutopie" (350), einem "Garten Erde" (350) und sogar von "Weltaufgang" (351) die Rede, wodurch die Hoffnung auf den "Beginn einer langen offenen und gestaltbaren Zukunft" (357) gemacht werden soll. Der elegische Ton des Katastrophennarrativs wird also durch ein eutopisches Narrativ ersetzt, wobei man sich fragen kann, ob damit die "Apokalypse-Angst” (Krause 2014) bereits überwunden ist. Solche gestalterischen Visionen, die aus kulturhistorischer Perspektive keineswegs neu sind, werden insbesondere im Wissenschaftsjournalismus reaktiviert, um eine positive Besetzung des Anthropozän-Konzepts zu lancieren und in politischer Hinsicht handlungsstimulierend für ein Umdenken in Richtung einer Großen Transformation zu wirken.

Die zweite, häufiger vorkommende Metapher ist die des "Raumschiffs Erde ohne Notausgang",12 für das in Rekurs auf Richard Buckminster Fullers bekanntes Buch Operating Manual for Spaceship Earth (1968) noch die Bedienungsanleitung gesucht wird. Gemäß dieser Metapher hängt die Sicherung des Überlebens davon $a b$, dass es ,uns' gelingt, "alternative Wege zu finden und die zukünftigen Folgen unseres Handelns zu antizipieren" (Bühler 2016: 159). Gleichwohl begünstigt diese Metapher im Unterschied zum Gärtner eine stark technologische Vorstellung für die Bewältigung der globalen Umweltkrise, die Berührungspunkte zum (bio-)technologischen Narrativ aufweist.

\subsection{Das (bio-)technologische Narrativ}

Das Narrativ der Großen Transformation findet einerseits seine Fortführung und Radikalisierung im (bio-)technologischen Narrativ, andererseits steht es auch im Kontrast dazu, da sich Vertreter der Großen Transformation von starken Eingriffen wie etwa dem Geoengineering und ökomodernistischen Träumen deutlich distanzieren. Doch Crutzen, Schwägerl, Ellis und andere Anthropozäniker

12 Vgl. die Essaysammlung Das Raumschiff Erde hat keinen Notausgang (2011); vgl. auch Sloterdijk 2015: 38-40; Malkmus 2016; Bühler 2016: 159, 184. 
stehen Formen des Biofuturismus und neuartigen (nicht nur grünen) Technologien durchaus positiv gegenüber. Das Geoengineering sieht die klein-, mittel- und großskalige Manipulation der Atmosphäre und der Biosphäre vor, etwa die Stimulierung des Planktonwachstums durch künstliche Erhöhung des Eisensulfatgehalts in den Ozeanen oder die Verminderung des Erderwärmungseffekts durch Einbringen von Schwefelsulfat in die Atmosphäre oder gar in die Stratosphäre (z.B. Crutzen 2006). Wegen möglicher nicht-intendierter Nebenwirkungen ist das Geoengineering jedoch heftig umstritten (z.B. Steffen et al. 2007: 620)..$^{13}$ Auch sehen etliche darin einen neo-prometheischen Umgang mit der Natur und die Gefahr einer fortgesetzten destruktiven Auswirkung auf die ökonomischen, sozialen und ökologischen Systeme (z.B. Rose et al. 2012: 2-5).

Das fortschrittsoptimistische Narrativ bestimmt auch die Idee einer "Grünen Revolution 2.0", welche die Probleme der Welternährung durch nachhaltige Intensivierung der Landwirtschaft, Protein-Ersatzstoffe und neue technisch hergestellte Lebensmittelformen lösen will. Ziel einer zweiten Grünen Revolution sind die Verbesserung von Ernteerträgen, Ernährungssicherheit und Armutsbekämpfung bei stark vermindertem Einsatz von Düngemitteln und intensivierter Landnutzung auf weniger Fläche, die vor allem den Landwirten in Entwicklungsländern zugutekommen soll, die proportional einen größeren Anteil von Arbeit in der Landwirtschaft haben (Lynch 2007: 494). Allerdings setze dies den Einsatz genetisch veränderten Saatguts (Soja, Weizen, Mais, Reis etc.) voraus, was durch Marktmacht weniger Firmen und einzelner Forschungsinstitute die Gefahr erneuter ökonomischer und sozialer Ungleichheit hervorbringen könnte (vgl. z.B. Pingali 2012).

Ähnlich ambivalent wird die "Ökobewegung 2.0" diskutiert, die insbesondere durch das "Ecomodernist Manifesto" (Asafu-Adjaye et al. 2015) hervorgetreten ist. ${ }^{14}$ Das 14-Punkte-Manifest ver-

13 Bemerkenswert ist, dass sich offenbar mittlerweile auch Crutzen etwas davon distanziert, wenn in einem gemeinsamen Artikel mit Will Steffen (et al. 2011: 858) eingeräumt wird: "Needless to say, the possibility of adverse environmental side effects must be fully researched before countermeasures to greenhouse warming are attempted." 14 In der Heuristik Bonneuils (2016: 23-25) wird lediglich der Aspekt des ,good Anthropocene' als "post nature grand narrative" bezeichnet. spricht „ein großartiges Anthropozän" (2. Abs.) mit Wohlstand für alle Menschen bei hoher Energieverfügbarkeit durch hocheffiziente Solar- und Kernenergie, aber niedrigem Ressourceneinsatz und verbessertem Naturschutz. Dort heißt es: „Die Intensivierung vieler menschlicher Aktivitäten insbesondere Landwirtschaft, Energiegewinnung, Forstwirtschaft und Besiedelung - um den Flächenverbrauch zu reduzieren und den Eingriff in die Natur zu verringern, ist der Schlüssel, um die menschliche Entwicklung von Umweltauswirkungen zu entkoppeln. Diese sozioökonomischen und technologischen Prozesse sind für die wirtschaftliche Modernisierung und den Umweltschutz von zentraler Bedeutung" (5. Abs.). Das Manifest mit 18 beteiligten AutorInnen, unter ihnen auch Erle Ellis als Vertreter eines, good Anthropocene', entstammt dem industrienahen US-amerikanischen Breakthrough Institute, deren Gründer Michael Shellenberger und Ted Nordhaus den "Death of Environmentalism" (2004) verkündet haben. Helmuth Trischler (2016a: 285) hat zu Recht kritisch dagegen eingewandt, dass mit solchen Ideen das Anthropozän-Konzept „für umstrittene wissenschaftliche Interessen instrumentalisiert" werde. Auch besteht die Befürchtung, "the contradiction between the spirit of emancipation and its catastrophic outcomes" aus dem Blick zu verlieren, sodass auch "unintended consequences" fahrlässig in Kauf genommen würden (Latour 2012). Im (bio-)technologischen Narrativ wird das Anthropozän strukturell als eine "Theodizee" bzw. in säkularisierter Variante als "anthropodicy" (Hamilton 2015: 233) konstruiert, welche das Übel in der Welt - wie etwa die Klimakrise und andere ökonomische, ökologische und soziale Verwundbarkeiten - durch eine (öko-)prometheische Steuerung der Gesellschaft mit technokratischen Mitteln für überwindbar erklärt.

In den letzten drei, vier Jahren lassen sich interessante Absetzbewegungen im breiten Lager der Anthropozäniker beobachten, wenn die einen insbesondere die Projekte des Geoengineering oder des Ökomodernismus als neoprometheische Hybris oder neuerlichen "Anthropologismus" (Schmieder 2014: 47) ablehnen, während die anderen technologische Innovationen für eine wichtige Stufe der Anpassung auf dem Weg zur Großen Transformation halten. Aussagekräftig ist die enorme Ernüchterung, die sich bei dem Philosophen Bernd Scherer, Intendant des "Anthro- 
pozän-Projekts" im Haus der Kulturen der Welt, einstellte, wenn er sich im Rückblick fragte, wie das "durchaus utopische Projekt [...] derart entgleisen" konnte, sei doch die Vorstellung vom Menschen als "Schöpfer einer neuen Erde", der als "Erfinder neuer Technologien [...] an die Stelle Gottes" trete, "alles andere als beruhigend" (Scherer 2015: 106). Diese Bedenken berühren Motive des Interdependenz-Narrativs, in dem angesichts der aufgelösten Natur-Kultur-Dichotomie nach einer Neubestimmung der Stellung des Menschen auf dem Planeten gesucht wird.

\subsection{Das Interdependenz-Narrativ}

Indem die Rede vom Menschen als geophysikalischer Kraft die Subjekt-Objekt-Dichotomie auf neuartige Weise infrage stellt, rückt das Anthropozän insbesondere als "kulturelles Konzept" (Trischler 2016a: 270) ins Zentrum der Aufmerksamkeit. Es ist Gegenstand verschiedener geistes- und kulturwissenschaftlicher Disziplinen sowie der Environmental Humanities, die seit ca. 2009 als „umbrella term" für die inter- und transdisziplinäre Vernetzung von Ecocriticism, Umweltgeschichte, Umweltphilosophie, Umweltethnologie, Kulturgeographie und Politische Ökologie (Heise 2013: 19) im Begriffe sind, sich mit eigenen Zeitschriften, Forschernetzwerken und Nachwuchsprogrammen zu etablieren (vgl. Wilke 2015).

Ursula Heise (2015: 40) sieht die Anthropozän-Idee als Chance, "den Menschen posthumanistisch neu zu denken" und ihn als ein "Teil von Netzwerken verteilter Handlungsträger" zu konzipieren, "die auch Tiere, Pflanzen, Substanzen und Gegenstände einschließen". Dieser Gedanke bezieht sich auf philosophische und wissenschaftstheoretische Debatten, die seit den frühen 1990er-Jahren die lange Zeit gültige Gegenüberstellung von Natur und Kultur problematisiert haben. Zu nennen sind hier v.a. die wichtigen Impulse, die von Donna Haraway, Bruno Latour, Rosi Braidotti und Cary Wolfe ausgegangen sind. Bei innen ist Natur nicht mehr als das Andere, als das Objekt wissenschaftlicher oder ästhetischer Anschauung konzipiert; stattdessen gehen sie von einem gegenseitigen Abhängigkeitsverhältnis von Mensch und Natur, ja sogar von einer Quasi-Interaktivität aus. Der Mensch ist demnach nicht als getrennt von anderen Spezies zu denken, sondern als Teil eines Netzwerkes im Austausch mit anderen Agenzien. Die Aufhebung der Subjekt-Objekt-Dichotomie bedeutet beispielsweise für Latour (2014: 17): "The point of living in the epoch of the Anthropocene is that all agents share the same shape-changing destiny". Das heißt für ihn aber nicht, dass Natur und Gesellschaft wiederversöhnt würden, sondern dass es vonnöten sei, dass die Agenzien so weit und differenziert wie möglich distribuiert werden. Vor dem Hintergrund eines kritischen Posthumanismus zeigt Braidotti (2016: 36f.), dass die Ablehnung der Artenhierarchie und die "Ent-Identifizierung mit der Menschheit" in einer globalisierten Welt Schwierigkeiten verschiedenster Art mit sich bringt, da eine neue "posthumane politische Ökonomie" dazu tendiere, "entscheidende Differenzen unsichtbar zu machen - insbesondere strukturelle Diskriminierung und Ungerechtigkeit". Eine posthumane kritische Theorie könne demgegenüber auf die wichtigen Differenzen aufmerksam machen, da das ,Wir', das in ein neues Zeitalter eingetreten ist, "nicht als homogene, geschweige denn universelle Einheit, sondern eher als nomadisches Gefüge" agiere (Braidotti 2016: 37).

Braidottis Ablehnung universaler Kategorien nimmt unter anderem Bezug auf eine Diskussion, die Dipesh Chakrabartys viel zitierter Essay Four Theses (2009) ausgelöst hat. Darin führt Chakrabarty die Konsequenzen der Idee einer kollektiv gedachten Menschheit als geophysikalischer Kraft aus, die sich im Zeichen der globalen Klimakrise herausgebildet habe: der Zusammenbruch der seit dem 17. Jahrhundert gezogenen Trennung von Natur- und Menschheitsgeschichte, die Infragestellung der Errungenschaften von Freiheit als Ergebnis von kultureller und historischer Vielfalt seit der Aufklärung, eine Koppelung der Speziesgeschichte mit der bestehenden kapitalistisch geprägten Fortschrittsgeschichte und schließlich die Grenzen historischen Verstehens überhaupt. Dabei hat er auch den streitbaren Begriff der "negativen Universalgeschichte" vorgeschlagen (Chakrabarty 2009: 222), da sich die Menschheit nicht mehr im Hegel'schen Sinne als eine aufgrund von rationalen Entscheidungen und historischen Veränderungen handelnde Allgemeinheit konzipieren lasse, sondern als ein Kollektiv erscheine, das zwar Dinge bewirke, ohne jedoch 
das Ausmaß des damit verbundenen Schreckens erfassen zu können. Die Kritik und Revision, die Chakrabartys Thesen fordern, zeigt die Notwendigkeit, den ontologischen Status des Menschen neu zu bestimmen, was die Frage nach den Konturen von wissenschaftlicher und menschlicher Erkenntnis einschließt, ohne die Erforschung disziplinärer und sozialer Differenzen außer Acht zu lassen (vgl. Emmet/Lekan 2016: 10; Ferrando 2016). Gegenüber einer planetarischen Perspektive heben Robert Emmet und Thomas Lekan (2016: 11) den Bedarf einer konkreten Politik der Verminderung der Auswirkungen des Klimawandels wie auch die Wichtigkeit von Utopien und Kreativität im täglichen Leben hervor, um unsere Möglichkeiten wie auch die Grenzen zu erkunden. Zentral für das Interdependenz-Narrativ ist dabei die Verknüpfung von geologischer Tiefenzeit und Menschheitsgeschichte (Dukes 2011; Davies 2016), welche nicht nur die Perspektive der Geschichtswissenschaften mit unterschiedlichen Periodisierungsnarrativen (vgl. Trischler 2016b: 312-317) erweitert, sondern auch neue Fragen für andere geistes-, kultur- und medienwissenschaftliche Disziplinen aufwirft.

Auch Latour geht von der Untrennbarkeit von Menschheits- und Erdgeschichte aus und hat dafür 2013 in seinen Gifford Lectures den Begriff der "geostories" eingeführt. Geostories gehen von keiner Intentionalität, sondern von einer den natürlichen Dingen innewohnenden Kraft, einer Agency, aus. Latour (2013: 74) definiert sie als „a form of narration inside which all the former props and passive agents have become active without, for that, being part of a giant plot written by some overseeing entity". Für inn hat demnach die gesamte Biosphäre eine Geschichte in dem Sinne, dass Veränderungen von Agenzien in der Umwelt eine Geschichte haben, der Bedeutung zukomme. Solche Agenzien sind aber keineswegs nur Fossilien, natürliche Ablagerungen oder Gesteins- und Landschaftsformationen, aus denen wir Umweltund Klimageschichte ablesen können. Der Begriff der geostories nimmt vielmehr einen neuen, state of nature' an, der aus, vielen Akteuren' besteht, zu denen etwa Kohlendioxid, Windturbinen, Atomkraft, Algen, Technofossilien oder Plastik gehören. Eine solche neomaterialistische Konzeption von ,NatureCultures' (Sullivan 2015) verlangt nach einer transhumanen, relationalen, netzwerkbezogenen Erforschung der natürlichen, kulturellen und gesellschaftlichen Umwelten (Braidotti 2016: 38); eine abstrakte Vorstellung von der Menschheit als kollektiver Akteur oder gar eine neoprometheische Konzeption des Menschen haben darin keinen Platz.

Eine kulturwissenschaftliche Differenzierung nehmen auch Eva Horn und Peter Schnyder (2016: 10) in ihrem Themenheft "Klimageschichte" der Zeitschrift für Kulturwissenschaften vor, in dem sie dafür plädieren, das Anthropozän nicht nur naturwissenschaftlich, sondern auch in seinen "historischen, [...] kulturellen und sozialen Dimensionen" zu betrachten. Dabei berufen sie sich auf Chakrabartys "doppelte Perspektive" einer Verbindung von Natur- und Menschheitsgeschichte und folgern daraus: "den anthropos [...] einerseits als biologische Spezies, andererseits aber auch als historisch, kulturell und politisch differenziert und heterogen wahrzunehmen" (10). Dabei sei es "nicht einfach die ,Spezies Mensch', deren industrielle Entwicklung sich verheerend auf das ökologische System des Planeten auswirkt - es sind vielmehr einzelne Kulturen, Technologien und Gesellschaften, die dies in sehr unterschiedlichem Maße und auch mit sehr unterschiedlichem Bewusstsein dafür tun" (10). Hier verbindet sich das Interdependenz-Narrativ mit Motiven des Gerichtsnarrativs, was einen sehr produktiven Strang in der gesamten geistes- und kulturwissenschaftlichen Forschung zum Anthropozän-Diskurs ausmacht.

Eine Erforschung des Anthropozän als kulturelles Konzept kann auch bedeuten, die "narrativen, philosophischen, ethischen und ästhetischen Dimensionen sichtbar, spürbar und moralisch anspornend zu machen" (Garrard et al. 2014: 150). Auch der Sammelband Readings in the Anthropocene (Wilke/Johnstone 2017) untersucht die Wechselbeziehungen zwischen der menschlichen und nicht-menschlichen Umwelt in Literatur, Philosophie, Geschichte und Kultur mittels einer Verbindung von Environmental Humanities, Germanistik und Kulturwissenschaft. In ähnlicher Weise stellt der Band German Ecocricitism in the Anthropocene (Sullivan/Schaumann 2017) die literatur- und kulturwissenschaftliche Analyse von deutschsprachigen Texten und Filmen vom 19. Jahrhundert bis heute in den Kontext von transnationalen, kosmopolitischen und planetarischen Perspektiven der ästhetischen Erkundung physischer Umwelten. Zudem werden neue Formen von Agency, die dezidiert nicht-menschliche 
Entitäten einschließen, in der poetischen Imagination thematisiert (vgl. Dürbeck et al. 2015). Der Amerikanist Hubert Zapf (2016) erforscht Literatur als Form der Kulturökologie, indem er zeigt, wie Sprache, Imagination und Kritik kulturelle Narrative von Mensch-Natur-Verhältnissen transformieren und damit zur Reflexion nachhaltiger Lebensformen beitragen.

Durch die unüberschaubare Komplexität der Zusammenhänge, welche das Anthropozän zum Ausdruck bringt, sehen manche insbesondere die Lyrik als geeignetes Medium an, "a sense of place" zu vermitteln (Bristow 2015: 6; vgl. auch Bayer/ Seel 2016; Solnick 2017). Mit Blick auf anglophone Romane zum Klimawandel und Anthropozän sieht Adam Trexler (2015: 5f.) die Aufgabe der Literaturwissenschaft darin, solche Texte als „kulturelle Texte" zu lesen, welche nicht nur emotionale und ästhetische Erfahrungen, sondern auch komplexe Netzwerke von Ideen darstellen, ohne die Komplexität von so "enormen kulturellen Transformationen wie dem Anthropozän" zu reduzieren. Demgegenüber betont der ökokritische Anglist Timothy Clark die Nicht-Repräsentierbarkeit des Anthropozän, das durch die Überkomplexität der systemischen Zusammenhänge und die Nicht-Kalkulierbarkeit der Folgen menschlichen Handelns gekennzeichnet sei und daher "a disconcerting de-politicization" (Clark 2015: 6f., 13) beinhalte. Er sieht somit den Ecocriticism in der Pflicht, sich dieser Komplexität zu stellen und sich mit neuen Repräsentationsformen und Rezeptionsverfahren jenseits bisheriger ökokritischer Kategorien auseinanderzusetzen. Die hier genannten Publikationen liefern darüber hinaus Ansätze, um eine Poetik des Anthropozän zu entwickeln und zugleich den neuen Stellenwert von Literatur- und Kulturwissenschaften, Ecocriticism, Kulturökologie und Environmental Humanities im interdisziplinären Anthropozän-Diskurs zu formulieren.

\section{Fazit und Ausblick: Pluralität und Metanarrativ der}

\section{Anthropozän-Narrative}

Im Anthropozän-Diskurs verwenden Wissenschafterlnnen narrative Strategien, um ihre Argumente und Interessen $\mathrm{zu}$ kommunizieren und breitere Resonanz zu erzeugen. Narrative Strategien werden auch zur kritischen Reflexion epistemologischer Voraussetzungen und etablierter Kategorien (z.B. Subjekt-Objekt-Dichotomie) eingesetzt. Im Anthropozän-Diskurs konkurrieren mehrere Narrative um Legitimität und Durchsetzung in den verschiedenen Wissenschaftsbereichen und Öffentlichkeiten. Dabei fungiert das Anthropozän zunächst als inter- und transdisziplinäres Brückenkonzept wie auch als Reflexionsbegriff (vgl. Dürbeck 2015). Das Spiel mit weiteren abgeleiteten Begriffsbildungen (vgl. Bonneuil/ Fressoz 2015; Haraway 2015, Morris et al. 2016) zeigt, wie sich das ursprünglich geologische Konzept verselbständigt hat und als kulturelles Konzept (Trischler $2016 \mathrm{a} / \mathrm{b}$ ) weiterentwickelt und in seiner Pluralität ausdifferenziert worden ist. Alternative Begriffsbildungen wie etwa Eurozän, Technozän, Kapitalozän oder Plantationocene können als Versuche verstanden werden, den Anthropozän-Diskurs hinsichtlich der Akteure in den westlichen Industriestaaten oder als konsequente Folge des kapitalistischen Systems oder neoliberaler Politiken zu spezifizieren. Die mit diesen Leitbegriffen verbundenen Theorien werden durchgehend in narrativer Form präsentiert oder zumindest durch Narrationen unterfüttert.

In den verschiedenen Anthropozän-Narrativen werden zum Teil stark divergierende politische, ökonomische, ethische und anthropologische Werthaltungen und Interessen artikuliert und oft gegensätzliche Handlungsorientierungen entworfen. Zugleich finden sich viele überlappende Elemente und Motive. ${ }^{15}$ Zudem weisen die verschiedenen Anthopozän-Narrative gemeinsame Strukturmerkmale auf, die auf einer strukturprägenden Ebene eine Verbindung herstellen, die über den Bezug auf das Leitkonzept des Anthropozän als gemeinsamen Nenner hinausgehen: erstens der Bezug auf die Gefährdung der Welt durch den Menschen als Plot; zweitens eine tiefenzeitliche Perspektive; drittens ein planetarischer Bezugsrahmen; viertens eine Aufhebung der kategorialen Grenzen zwischen Natur und

15 Helmuth Trischler (2016a: 283) spricht von einer „Vielzahl von sich teils überlappenden, teils konkurrierenden Narrativen und Weltbildern", deren "einziger gemeinsamer Nenner" die "Reintegration des Nicht-Menschlichen" in die historischen Erzählungen und anthropologischen Ontologien sei (vgl. auch Trischler 2016b: 328). 
Kultur im Horizont des Erdsystemkonzepts; fünftens die Thematisierung der ethischen Verantwortung für die Verminderung weiterer Umweltzerstörung und das Überleben der menschlichen Zivilisationen.

Das Vorliegen dieser gemeinsamen narrativen Strukturmerkmale bei allen fünf Anthropozän-Narrativen legt es nahe, von einem Metanarrativ des Anthropozän zu sprechen. Dieses metanarrative Erzählmuster wird in den fünf Anthropozän-Narrativen in unterschiedliche Settings übersetzt und entsprechend den unterschiedlichen Interessen variiert. Dabei unterscheiden sich die Narrative vor allem hinsichtlich der jeweiligen Figuren, die den Plot (mit seiner tiefenzeitlich-planetarischen Erdsystemperspektive) bevölkern. Zugleich divergieren die Protagonisten und deren Rollenverteilung zum Teil erheblich. Zum Beispiel wird einerseits die Menschheit als rettende Akteurin präsentiert, andererseits diese Idee eines universellen Akteurs als Verschleierungstaktik einer korrumpierten Elite zurückgewiesen. Pointiert ausgedrückt: Während die unterschiedlichen Anthropozän-Narrative hinsichtlich der meisten narrativen Dimensionen weitgehend übereinstimmen, unterscheiden sie sich zum Teil radikal hinsichtlich der Protagonisten und ihrer Rollen.

Die hier vorgestellte Unterscheidung von fünf Anthropozän-Narrativen versteht sich als heuristischer Ordnungsvorschlag. Sie überschneidet sich teilweise mit Bonneuils Unterscheidung von vier Narrativen, in deren Pluralität er die Möglichkeit der Reflexion und Repolitisierung des Anthropozän-Diskurses sieht (vgl. Bonneuil 2016: 29). Auch im vorliegenden Beitrag werden unterschiedliche Plots, deren narrative Strategien und Legitimationsfunktionen bzw. deren reflexive Kraft (wie beim Interdependenz-Narrativ) in den Vordergrund gestellt. Doch werden diese bei aller Pluralität auf gemeinsame Strukturmerkmale eines Metanarrativs bezogen. Neben der Pluralisierung der Anthropozän-Narrative, die nebeneinander bestehen, wird dadurch zugleich auf eine breite Anschlussfähigkeit und generative Kraft des Metanarrativs des Anthropozän hingewiesen. Aufbauend auf diesen Befunden lässt sich feststellen, dass die Narrative des Anthropozän Brückenschläge zwischen verschiedensten wissenschaftliche Disziplinen ermöglichen, die sich in dem ausgewerteten Textmaterial auf- fallend häufig finden: Geo- und UmweltwissenschaftlerInnen publizieren mit HistorikerInnnen und drängen in das Feld der Ethik und Politik oder werden zu ,Kulturwissenschaftlern' (Malkmus 2015: 196), Wissenssoziologen wie Latour (2013) sehen eine neue "politische Theologie der Natur", Kulturtheoretiker wie Sloterdijk (2015: 43) fordern eine "politische Anthropologie", Philosophen wie Bernd Scherer (2015: 240) plädieren für eine "sinnlich-ästhetische Praxis", Geschichts-, Literatur- und KulturwissenschaftlerInnen wie Chakrabarty, Heise, Horn oder Schnyder schließlich mahnen eine posthumane Selbstreflexion in erdgeschichtlicher Perspektive an, die den Menschen gleichwohl historisch, kulturell und politisch differenziert wahrnimmt.

Durch die narrative Struktur erzeugen markante Periodisierungen interdisziplinäre Verbindungen. So korrespondieren die beiden jüngeren Marker für den Beginn des neuen Erdzeitalters (um 1784; um 1950) mit zwei etablierten historischen Epochenbezeichnungen, nämlich dem Industrie- und dem Atomzeitalter (vgl. Schmieder 2014: 45), wodurch die Geologie auf Modelle der Geistes- und Sozialwissenschaften rekurriert. Indem sie sich diese aneignet und mit ihren Methoden und Daten untersucht, findet ein interund transdisziplinärer Umgang mit Epochenkategorien statt. Umgekehrt sehen die Geschichtsund die Kulturwissenschaften die Notwendigkeit, die Zivilisationsgeschichte mit der geologischen Tiefenzeit und Speziesgeschichte zu verbinden (z.B. Chakrabarty 2009), wodurch die tiefenzeitliche Perspektive ins Zentrum der zeitgenössischen Umweltpolitik rückt und das Verständnis der gegenwärtigen Umweltzerstörung und Ungleichheit verändert (vgl. Davies 2016). Es kommt aber auch zum Unbehagen an bestimmten narrativen Mustern, wenn Jan Zalasiewicz (2015: 177) bemerkt, der Zusammenfall des angenommenen Beginns des Anthropozän mit dem Atomzeitalter sei "nicht ganz ideal”, da dieses "mit einem Symbol des Weltuntergangs" belegt sei; damit distanziert er sich vom Katastrophennarrativ.

Aufbauend auf der hier vorgestellten Analyse stellen sich mehrere Forschungsfragen. Erstens wäre zu untersuchen, welche Strategien im Einzelnen zur Herausbildung so unterschiedlicher Narrative des Anthropozän beigetragen haben und auf welche Topoi und Erzähltraditionen (z.B. Modernenarrativ) dabei zurückgegriffen wird. 
Zweitens stellt sich die Frage, welche Effekte die Spannung zwischen pessimistischen und optimistisch-pragmatischen Ausgestaltungen des Metanarrativs hat, wenn es einerseits dystopische und warnend-appellative, andererseits eutopische und gar neoprometheische Erzählungen beinhaltet. Betrachtet man das Anthropozän als ein Metanarrativ, stellt sich zudem die Frage nach einem möglichen epistemologischen Totalitätsanspruch. Manche kritisieren beispielsweise das Anthropozän-Konzept als ein "hegemonic system" und sprechen zu Recht von einer "geocratic power" der Experten (Bonneuil/Fressoz 2015: 48,93 ) oder warnen vor der Vermessenheit einer "anthropodicy" (Hamilton 2015: 233). Aber man könnte das Metanarrativ auch als emergierende Struktur sehen, die aus den einzelnen Anthropozän-Narrativen hervorgeht und insofern keinen universalisierenden Anspruch hegt. Drittens ist zu fragen, ob die Vielzahl der Narrative und die Spannung zwischen ihnen die Sinnhaftigkeit des Anthropozän-Konzepts stabilisiert oder unterminiert; es könnte sein, dass sich die einzelnen Narrative soweit verselbständigen, dass sie ihre Bindungskraft zum geologischen wie auch zum kulturellen Anthropozän-Konzept verlieren, wenn es nicht überhaupt zum „vulgärwissenschaftlichen Topos" verkommt (Paál 2015: 7). Viertens stellt sich die Frage, ob es eine Korrelation zwischen der jeweiligen Periodisierung des Anthropozän und einzelnen Narrativen gibt, sodass die Erzählungen die Datierung des Beginns des neuen Zeitalters strategisch einsetzen. Abschließend lässt sich sagen, dass die inter- und transdisziplinäre Diskussion, die sich in den zum Teil gegenläufigen Narrativen des Anthropozän schon jetzt herausgebildet hat, wichtige Anstöße für neue Formen der disziplinüberschreitenden Zusammenarbeit zwischen Natur-, Sozial-, Geistes- und Kulturwissenschaften gibt, deren strukturierte Weiterentwicklung zukünftiger Forschung vorbehalten sein wird.

\section{Literatur}

Althor, Glenn/Watson, James E. M./Fuller, Richard A. (2016): Global Mismatch Between Greenhouse Gas Emissions and the Burden of Climate Change. In: Scientific Reports (published online), https://www. nature.com/articles/srep20281, S. 1-6 (abgerufen am 29.06.2017).
Assmann, Aleida (2016): Die Grenzenlosigkeit der Kulturwissenschaften. In: Kulturwissenschaftliche Zeitschrift 1.1, Sonderheft: Neue Horizonte der Kulturwissenschaft/en, S. 39-48.

Balter, Michael (2013): Archaeologists Say the ,Anthropocene' Is Here - But It Began Long Ago. In: Science 340/6130, S. 261-262.

Bayer, Anja/Seel, Daniela (Hg.) (2016): „all dies hier, Majestät, ist deins": Lyrik im Anthropozän. Anthologie. Berlin: kookbooks.

Becker-Lindenthal, Hjördis (2017): Sokrates im Anthropozän. Gedanken zum Menschenmöglichen im Ausgang von Blumenberg und Valéry. In: Valdivia Orozco, Pablo/Allerkamp, Andrea (Hg.): Paul Valéry: Für eine Epistemologie der Potentialität. Heidelberg: Winter, S. 155-173.

Boes, Tobias/Marshall, Kate (2014): Writing the Anthropocene. An Introduction. In: Minnesota Review 83, S. 60-72.

Böhme, Gernot (1989): Für eine ökologische Naturästhetik. Frankfurt a.M.: Edition Suhrkamp.

Bonneuil, Christophe/Fressoz, Jean-Baptiste (2015): The Shock of the Anthropocene. The Earth, History and Us. New York et al.: Verso Books.

Bonneuil, Christophe (2016): The Geological Turn. Narratives of the Anthropocene. In: Hamilton, Clive/ Bonneuil, Christophe/Gemenne, François (Hg.): The Anthropocene and the Global Environmental Crisis: Rethinking Modernity. London: Routledge, S. 15-31.

Braidotti, Rosi (2016): Jenseits des Menschen: Posthumanismus. In: Aus Politik und Zeitgeschichte 66, H. 37-38, S. 33-38.

Braungart, Georg (2009): Poetik der Natur. Literatur und Geologie. In: Anz, Thomas (Hg.): Natur - Kultur. Zur Anthropologie von Sprache und Literatur. Paderborn: Mentis, S. 55-77.

Bristow, Tom (2015): The Anthropocene Lyric. An Affective Geography of Poetry, Person, Place. Basingstoke: Palgrave Macmillan.

Bühler, Benjamin (2016): Ecocriticism. Grundlagen - Theorien - Interpretationen. Eine Einführung. Stuttgart: Metzler.

Chakrabarty, Dipesh (2009): The Climate of History: Four Theses. In: Critical Inquiry 35, S. 197-222.

Chakrabarty, Dipesh (2015): „Ein gemeinsame, aber differenzierte Verantwortung". Dipesh Chakrabarty im Gespräch mit Katrin Klingan. In: Renn, Jürgen/Scherer, Bernd (Hg.): Das Anthropozän. Zum Stand der Dinge. Berlin: Matthes \& Seitz, S. 142-159.

Clark, Timothy (2015): Ecocriticism on the Edge. The Anthropocene as Treshold Concept. London et al.: Bloombury Academic.

Colebrook, Claire (2014): Death of the PostHuman. Essays on Extinction. 1. Bd. Ann Arbor: Open Humanities Press.

Cronon, William (1992): A Place for Stories: Nature, History, and Narrative. In: Journal of American History, S. $1347-1376$

Crutzen, Paul (2002): Geology of Mankind. In: Nature 415, S. 23. 
Crutzen Paul J. (2006): Albedo Enhancement by stratospheric Sulfur Injections: A Contribution to Resolve a Policy Dilemma. In: Climate Change 77, S. 211-219.

Crutzen, Paul/Schwägerl, Christian (2011): Living in the Anthropocene: Toward a New Global Ethos. In: Yale Environment 360 (24. 01. 2011), http://e360.yale. edu/features/living_in_the_anthropocene_toward_a_ new_global_ethos (abgerufen am 29.06.2017).

Crutzen, Paul Josef/Steffen, Will (2003): How Long Have We Been in the Anthropocene Era? In: Climatic Change 61.3, S. 251-257.

Crutzen, Paul/Stoermer, Eugene F. (2000): The ,Anthropocene'. In: Global Change Newsletter 41, S. 17-18.

Davies, Jeremy (2016): The Birth of the Anthropocene. Oakland: University of California Press.

Dibley, Ben (2012): Ecological Humanities. ,The Shape of Things to Come'. Seven Theses on the Anthropocene and Attachment. In: Australian Humanities Review 52, http://www.australianhumanitiesreview. org/archive/Issue-May-2012/dibley.html (abgerufen am 27.06.2017)

Dürbeck, Gabriele (2015): Das Anthropozän in geistesund kulturwissenschaftlicher Perspektive. In: Dürbeck, Gabriele/Stobbe, Urte (Hg.): Ecocriticism. Eine Einführung. Köln: Böhlau, S. 107-119.

Dürbeck, Gabriele/Schaumann, Caroline/Sullivan, Heather (2015): Human and Non-human Agencies in the Anthropocene. In: Ecozona. European Journal of Literature, Culture and Environment 3.1, S. 118-136.

Dukes, Paul (2011): Minutes to Midnight: History and the Anthropocene Era from 1763. London et al: Anthem Press.

Ellis, Erle, C. (2011): The Planet of no Return. Human Resilience on an Artificial Earth. In: Breakthrough Journal 2, http://breakthroughjournal.org/content/ authors/erle-ellis/the-planet-of-no-return/shtml (abgerufen am 25.06.2017).

Ellis, Erle C./Ramankutty, Navin (2008): Putting People in the Map: Anthropogenic Biomes of the World. In: Frontiers in Ecology and the Environment 6, S. 439447.

Emmet, Robert/Lekan, Thomas (2016): Introduction. In: RCC Perspectives. Transformations in Environment and Society 2, Themenheft: Whose Anthropocene? Revisiting Dipesh Chakrabarty's „Four Theses", S. 7-11.

Erll, Astrid/Roggendorf, Simone (2002): Kulturgeschichtlich Narratologie: Die Historisierung und Kontextualisierung kultureller Narrative. In: Nünning, Ansgar/ Nünning, Vera: Neue Ansätze in der Erzähltheorie. Trier. Wissenschaftlicher Verlag, S. 73-113.

Feindt, Peter H. (2002): Gemeinsam gegen Niemanden. Der Umwelt- und Nachhaltigkeitsdiskurs in Deutschland. In: Neue Soziale Bewegungen 4, Themenheft: Etablierte Herausforderer? Akteure und Diskurse der Umweltpolitik, S. 20-28.

Ferrando, Francesca (2016): The Party of the Anthropocene: Post-humanism, Environmentalism and the Post-anthropocentric Paradigm Shift. In: Relations. Post-Anthropocentrism 4.2, S. 159-173.
Fisher, Walter (1984): Narration as Human Communication Paradigm: The Case of Public Moral Argument. In: Communication Monographs 51, S. 1-22.

Fuller, Richard Buckminster (1968): Operating Manual for Spaceship Earth. Carbondale: Southern Illinois University Press.

Garrard, Greg/Handwerk, Gary/Wilke, Sabine (2014): Introduction: Imagining Anew: Challenges of Representing the Anthropocene. In: Environmental Humanities 5, S. 149-153.

Hamann, Alexandra/Leinfelder, Reinhold/Zea-Schmidt, Claudia (2013): Die große Transformation. Klima Kriegen wir die Kurve? Berlin: Jacoby \& Stuart.

Hamilton, Clive (2015): The Theodicy of the ,Good Anthropocene'. In: Environmental Humanities 7, S. 233-238.

Hamilton, Clive/Grinevald, Jacques (2015): Was the Anthropocene anticipated? In: The Anthropocene Review 2.1, S. 59-72.

Haraway, Donna (2003): The Companion Species Manifesto. Dogs, People, and Significant Otherness. Chicago: University of Chicago Press.

Haraway, Donna (2015): Anthropocene, Capitalocene, Plantationocene, Chthulucene: Making Kin. In: Environmental Humanities 6, S. 159-165.

Heise, Ursula K. (2013): Comparative Ecocriticism in the Anthropocene. In: Komparatistik. Jahrbuch der Deutschen Gesellschaft für Allgemeine und Vergleichende Literaturwissenschaft, S. 19-30.

Heise, Ursula K. (2015): Posthumanismus. Den Menschen neu denken. In: Möllers, Nina et al. (Hg.): Willkommen im Anthropozän. Unsere Verantwortung für die Zukunft der Erde. München: Deutsches Museum Verlag, S. 38-42.

Heise, Ursula K. (2016): Imagening Extinction: The Cultural Meanings of Endangered Species. Chicago: University of Chicago Press.

Horn, Eva/Schnyder, Peter (2016): Romantische Klimatologie. In: Zeitschrift für Kulturwissenschaften H. 1, S. 9-18.

James, Erin (2015): The Storyworld Accord: Econarratology and Postcolonial Narratives. Lincoln/London: University of Nebraska Press.

Kolbert, Elizabeth (2015): The Sixth Extinction. An Unnatural History. London: Bloomsbury.

Koschorke, Albrecht (2012): Wahrheit und Erfindung. Grundzüge einer Allgemeinen Erzähltheorie. Frankfurt a. M.: S. Fischer.

Latour, Bruno (2012): Love Your Monsters. Why we must Care for Our Technologies as We Do Our Children. In: Breakthrough Journal 2, http://breakthrough. org/index.php/journal/past-issues/issue-2/love-yourmonsters (abgerufen am 25.06.2017).

Latour, Bruno (2013): Facing Gaia. Six Lectures of the Political Theology of Nature (Gifford Lectures). http://www.bruno-latour.fr/sites/default/files/downloads/GIFFORD-ASSEMBLED.pdf (abgerufen am 30.09.2016).

Latour, Bruno (2014): Agency at the Time of the Anthropocene. In: New Literary History 45, 1-18. 
Leggewie, Claus/Welzer, Harald (2009): Das Ende der Welt, wie wir sie kannten. Klima, Zukunft und die Chancen der Demokratie. Frankfurt a.M.: S. Fischer.

Leinfelder, Reinhold/Schwägerl, Christian/Möllers, Nina et al. (2012): Die menschengemachte Erde. Das Anthropozän sprengt die Grenzen von Natur, Kultur und Technik. In: Kultur und Technik 2, S. 12-17.

Leinfelder, Reinhold (2015): ,Die Zukunft war früher auch besser' - Neue Herausforderungen für die Wissenschaft und ihre Kommunikation. In: Möllers, Nina et al. (Hg.): Willkommen im Anthropozän. Unsere Verantwortung für die Zukunft der Erde. München: Deutsches Museum Verlag, S. 97-102.

Lewis, Simon L./Maslin, Mark A. (2015): Defining the Anthropocene. In: Nature 519, S. 171-180.

Lynch, Jonathan P. (2007): Roots of the Second Green Revolution. In: Australian Journal of Botany 55, S. 493-512.

MacIntyre, Alasdair C. (1984): After Virtue. A Study in Moral Theory. 2. Aufl. Notre Dame: University of Notre Dame Press.

Malkmus, Bernhard (2015): Naturgeschichten vom Fisch, oder: Die Angst vor dem Anthropozän. In: Scheideweg. Jahresschrift für skeptisches Denken, S. 183200.

Malm, Andreas/Hornborg, Alf (2014): The Geology of Mankind? A Critique of the Anthropocene Narrative. In: The Anthropocene Review 1.1, S. 62-66.

Manemann, Jürgen (2014): Kritik des Anthropozäns. Plädoyer für eine neue Humanökologie. Bielefeld: transcript.

Masco, Joseph (2010): Bad Weather: On Planetary Crisis. In: Social Studies of Science 40.1, S. 7-40.

Mastrandrea, Michael, D./Schneider, Stephen H. (2011): Vorbereitungen für den Klimawandel. In: Das Raumschiff hat keinen Notausgang. Energie und Politik im Anthropozän. Frankfurt a.M.: Suhrkamp, S. 11-59.

McKibben, Bill (1989): The End of Nature. New York: Anchor.

Morton, Timothy (2014): How I Learned to Stop Worrying and Love the Term Anthropocene. In: The Cambridge Journal of Postcolonial Literary Inquiry 1/2, S. 257-264.

Neumann, Birgit (2013): Narrativistische Ansätze. In: Nünning, Ansgar (Hg.): Metzler Lexikon Literaturund Kulturtheorie. Ansätze - Personen - Grundbegriffe. 5. Aufl. Stuttgart: Metzler, S. 552-555.

Nietzsche, Friedrich (2003): Also sprach Zarathustra II: Von großen Ereignissen. In: ders: Werke. Kritische Gesamtausgabe. Hg. von Giorgio Colli und Mazzino Montinari. 1. Bd., 6. Abt. München: dtv.

Moore, Jason W. (Hg.) (2016): Anthropocene of Capitalocene? Nature, History, and the Crisis of Capitalism. Oakland/CA: PM Press.

Oreskes, Naomi/Conway, Erik M. (2014): The Collapse of the Western Civilization. A View from the Future. New York: Columbia University Press.

Paál, Gábor (2015): Das Anthropozän muss wissenschaftlich bleiben. In: Spektrum der Wissenschaft 20, S. 6-10.
Parikka, Jussi (2015): A Geology of Media. Minneapolis/ London: University of Minnesota Press.

Pingali, Prabhu L. (2012): Green Revolution: Impacts, Limits, and the Path Ahead. In: Proceedings of the National Academy of Sciences of the United States of America (PNAS) 109, Nr. 31, S. 12302-12308.

Renn, Jürgen/Scherer, Bernd (2015): Einführung. In: dies. (Hgg.): Das Anthropozän. Zum Stand der Dinge. Berlin: Matthes \& Seitz, S. 7-23.

Revkin, Andrew (1992): Global Warming. Understanding the Forecast. New York: Abbeville.

Rockstroem, Johan/Steffen, Will et al. (2009): A Safe Operating Space for Humanity. In: Nature 461, S. $472-475$.

Rose, Deborah Bird/ van Dooren, Thom/Chrulew, Matthew et al. (2012): Thinking Through the Environmental, Unsettling the Humanities. In: Environmental Humanities 1, S. 1-5.

Schaumann, Caroline/Sullivan, Heather (Hgg.) (2017): German Ecocriticism in the Anthropocene. New York: Palgrave Macmillan.

Schellnhuber, Hans Joachim/Huber, Veronika (2013): Melting and Mystification. A Comparative Analysis of Mitigation and Adaptation Strategies. In: Crutzen, Paul J. et al. (Hgg.): Fate of Mountain Glaciers in the Anthropocene. Vatican: City: Pontifical Academy of Sciences, S. 1-18.

Scherer, Bernd M. (2015): Der blinde Fleck der Aufklärung. Zum Verständnis von Natur und Kultur. In: Möllers, Nina et al. (Hgg.): Willkommen im Anthropozän. Unsere Verantwortung für die Zukunft der Erde. München: Deutsches Museum Verlag, S. 103-107.

Scherer, Bernd (2015): Die Monster. In: Renn, Jürgen/ Scherer, Bernd (Hgg.): Das Anthropozän. Zum Stand der Dinge. Berlin: Matthes \& Seitz, S. 241-226.

Schmieder, Falko (2014): Urgeschichte der Nachmoderne. Zur Archäologie des Anthropozäns. In: Forum Interdisziplinäre Begriffsgeschichte [E-Journal], 3.2, S. 43-48.

Schopenhauer, Arthur (1973): Die Welt als Wille und Vorstellung. In: ders.: Sämtliche Werke. Textkritisch bearbeitet. Hg. von Wolfgang Freiherr von Löhneysen. Bd. II. Frankfurt a.M: Suhrkamp.

Schwägerl, Christian (2012): Menschenzeit. Zerstören oder Gestalten? Wie wir heute die Welt von morgen erschaffen. München: Goldmann.

Sloterdijk, Peter (2015): Das Anthropozän - ein ProzessZustand am Rand der Erd-Geschichte? In: Renn, Jürgen/Scherer, Bernd (Hgg.): Das Anthropozän. Zum Stand der Dinge. Berlin: Matthes \& Seitz, S. 25-44.

Solnick, Sam (2017): Poetry and the Anthropocene. Ecology, Biology and Technology in Contemporary British and Irish Poetry. London: Routledge.

Steffen Will/Crutzen, Paul J./McNeill, John R. (2007): The Anthropocene: Are Humans Now Overwhelming the Great Forces of Nature? In: Ambio 36.8, S. 614-621.

Steffen, Will/Grinevald, Jacques/Crutzen, Paul/McNeill, John (2011): The Anthropocene: Conceptual and Historical Perspectives. In: Philosophical Transactions of the Royal Society A 369, S. 842-867. 
Steffen, Will/Broadgate, Wendy/Deutsch, Lisa et al. (2015): The Trajectory of the Anthropocene: The Great Acceleration. In: The Anthropocene Review, S. 81-98.

Stoppani, Antonio (1873): Corso di geologia. Volume II Geologia stratigrafica. Mailand: Bernardoni.

Sullivan, Heather (2015): New Materialism. In: Dürbeck, Gabriele/Stobbe, Urte (Hgg.): Ecocriticism. Eine Einführung. Köln: Böhlau, S. 57-67.

Trexler, Adam (2015): Anthropocene Fictions. The Novel in a Time of Climate Change. Charlottesville/London: University of Virginia Press.

Trischler, Helmuth (2016a): Zwischen Geologie und Kultur: Die Debatte um das Anthropozän. In: Bayer, Anja/ Seel, Daniela (Hgg.): „all dies hier, Majestät, ist deins": Lyrik im Anthropozän. Anthologie. Berlin: kookbooks, S. 269-286.

Trischler, Helmuth (2016b): The Anthropocene. A Challenge for the History of Science, Technology, and the Environment. In: N.T.M. - Journal of the History of Science, Technology, and Medicine 24/3, S. 309-335.

Watkin (2015): Michel Serres' Great Story: From Biosemiotics to Econarratology. In: SubStance 44.3, S. 171-187.

Wilke, Sabine (2015): Environmental Humanities. In: Dürbeck, Gabriele/Stobbe, Urte (Hgg.): Ecocriticism. Eine Einführung. Köln: Böhlau, S. 94-106.

Wilke, Sabine/Johnstone, Japhet (Hgg.) (2017): Readings in the Anthropocene. The Environmental Humanities, German Studies, and Beyond. New York et al.: Bloomsbury.

Zalasiewicz, Jan (2008): The Earth After Us - What Legacy will Humans Leave in the Rocks? New York: Oxford University Press.

Zalasiewicz, Jan/Williams, Mark/Smith, Alan et al. (2008): Are we now living in the Anthropocene? In: GSA Today 19.02.2008, S. 4-8.

Zalasiewicz, Jan/Williams, Mark/Steffen, Will/Crutzen, Paul Josef (2010): The New World of the Anthropocene. The Anthropocene, Following the Lost World of the Holocene, Holds Challenges for Both Science and Society. In: Environmental Science \& Technology 44.7 , S. $2228-2231$.

Zalasiewicz, Jan/Williams, Mark/Haywood, Alan/Ellis, Michael (2011): The Anthropocene: A New Epoch of Geological Time? In: Philosophical Transactions of the Royal Society A 369, Nr. 1938, S. 835-841.

Zalasiewicz, Jan (2015): Die Einstiegsfrage: Wann hat das Anthropozän begonnen? In: Renn, Jürgen/Scherer, Bernd (Hgg.): Das Anthropozän. Zum Stand der Dinge. Berlin: Matthes \& Seitz, S. 160-180.

Zapf, Hubert (2016): Literature as Cultural Ecology. Sustainable Texts. London et al.: Bloomsbury.

\section{Weitere Literatur}

Asafu-Adjaye, John/Blomqvist, Linus/Brand, Stewart et al. (2015): Ökomodernes Manifest, http://www.ecomodernism.org/deutsch/ (abgerufen am 30.06.2017).

Carrington, Damian (2016): The Anthropocene epoch: scientists declare dawn of human-influenced age. In: The Guardian, 29.08.2016, https://www.theguardian. com/environment/2016/aug/29/declare-anthropocene-epoch-experts-urge-geological-congress-human-impact-earth (abgerufen am 23.06.2017)

The Economist (26.05.2011): Welcome to the Anthropocene. Humans have changed the way the world works. Now they have to change the way they think about it, too. http://www.economist.com/ node/18744401 (abgerufen am 27.06.2017).

Häntzschel, Jörg/Lorch, Catrin/Menden, Alexander (2014): Aus den Fugen. Ist die Welt noch zu retten? Bestürzt stellen Wissenschaftler das Ausmaß fest, in dem der Mensch zur Naturgewalt geworden ist. Auf einer Tagung in Berlin verbündeten sich jetzt Wissenschaft und Kunst, um es zu begreifen. In: Süddeutsche Zeitung, 26.10.2014.

Krause, Norbert (2014): Debatte „Menschenzeit" - Das Ende der Apokalypse-Angst? Der Journalist Christian Schwägerl plädiert für ein „Anthropozän", http:// www. boell-thueringen.de/de/2014/03/12/menschenzeit-das-ende-der-apokalypse-angst (abgerufen am 30.05.2017).

Leinfelder, Reinhold (2013): Wir Weltgärtner. In dieser Woche wird in Berlin eine neue erdgeschichtliche Epoche eingeläutet: Das Anthropozän. Der Begriff soll unser Denken verändern. Ein Gespräch mit dem Geobiologen Reinhold Leinfelder von Ulrich Schnabel. In: DIE ZEIT 3, 10.01.2013, S. 32.

Rauchhaupt, UIf von (2015): Wie kommen wir nun ins Anthropozän? Unsere Spezies greift inzwischen derart tief in Erdprozesse ein, dass man überlegt, ein neues geologisches Zeitalter einzuführen. Dass das ist gar nicht so einfach. In: Frankfurter Allgemeine Sonntagszeitung, 31.05.2015, S. 58-59.

WBGU (2011): Welt im Wandel Gesellschaftsvertrag für eine große Transformation. Berlin: WBGU 2011. http://www.wbgu.de/hauptgutachten/hg-2011-transformation (abgerufen am 29.06. 2017).

Wenzel, Uwe Justus (2013): Ein neues Erdzeitalter. Verschwinden wir im Anthropozän? In: Neue Zürcher Zeitung, 14.01.2013. 